Теорія Ймовір. та Матем. Статист. Вип. 81, 2010
Theor. Probability and Math. Statist.

No. 81, 2010, Pages 177-186

S 0094-9000(2011)00818-6

Article electronically published on January 20, 2011

\title{
ARBITRAGE IN A DISCRETE TIME MODEL OF A FINANCIAL MARKET WITH A TAXATION PROPORTIONAL TO THE PORTFOLIO SIZE
}

UDC 519.21

\author{
G. M. SHEVCHENKO
}

\begin{abstract}
We introduce the notion of $V^{\varepsilon}$-arbitrage (in other words, an arbitrage under the taxation proportional to the portfolio size) for a multiperiod discrete time model of a financial market. For a $V^{\varepsilon}$-arbitrage, we prove a result analogous to the classical fundamental asset pricing theorem. Differences between a $V^{\varepsilon}$-arbitrage and some other notions of arbitrage are analyzed.
\end{abstract}

\section{INTRODUCTION}

A fundamental question arising in every mathematical model of a financial market is whether or not an arbitrage strategy exists for this model. The most important result on a relationship between the nonexistence of an arbitrage strategy and the existence of an equivalent martingale measure is proved in [1. Other proofs of this result as well as other equivalent statements are given in [2, 6.

Why is this notion so important and why do we always assume that models are arbitrage-free? The most popular explanation is that if arbitrage strategies exist, then the demand for arbitrage securities increases; that is, investors could attain infinite wealth by taking unlimited positions in those securities. Thus an arbitrage strategy leads to an increasing demand for some assets and a decreasing demand for some others, which should finally balance the prices at a reasonable level. The modern studies of the arbitrage problem show, however, that the existence of an arbitrage does not necessarily mean that the demand for it will be sufficiently high. This conclusion seems to be absurd at first glance. Below we try to clarify it.

Arbitrage strategies often lead to unlimited positions, which create a problem, especially when they are negative. The simplest illustration here is a martingale betting (double the bet each time you loose when flipping a coin). This strategy, being in fact an arbitrage one, leads to the quick increase of bets. After several applications, this martingale strategy leads to the ruin, since a next bet could be out of money.

Liu and Longstaff [7] consider an arbitrage model of a financial market and impose constraints on risky positions in a portfolio. It turns out that the textbook strategy (invest all the money in the arbitrage) becomes wrong and nonprofitable. Another conclusion made in [7] is that the Sharp ratio (profit/risk ratio) is rather small for the arbitrage strategy, and an optimal strategy is to invest a considerably less amount of money to

2010 Mathematics Subject Classification. Primary 91B30.

Key words and phrases. Arbitrage, transaction costs, portfolio size constraints, martingale measure, measurable choice theorem. 
the arbitrage as compared to the portfolio constraints. It is sometimes the case that investors should even avoid the arbitrage strategy at all.

The problems described above increase interest in the models containing portfolio constraints as well as transaction costs (which means that investors are forced to avoid big positions in their portfolio). The literature concerning the markets with transaction costs is rather extensive; see, for example, the paper [6], which contains the most important results and a survey of the relevant literature on the topic.

We consider another model involving a taxation proportional to the portfolio size instead of a model with transaction costs. The taxation is also a natural limiting factor that urges investors to decrease the portfolio size. We continue investigations initiated in [9]. Our approach differs from that in [10], where the portfolio constraints are fixed in advance and are not related to tax regulations.

The paper is organized as follows. In Section 1] we provide necessary definitions. Oneperiod models are studied in Section 2, where we recall some results of the paper [10] and state their counterparts for models with proportional taxation. The main result on the relationship between the nonexistence of an arbitrage and the existence of an equivalent martingale measure (which is an "almost" martingale measure) is given in Section 3 . An important auxiliary result on the joint measurability of the regular expectation is stated and proved in Section 4, this result is of its own interest.

\section{MAin NOTATiON}

Let $(\Omega, \mathcal{F}, \mathrm{P})$ be a complete probability space equipped with a filtration

$$
\left(\mathcal{F}_{t}\right)_{t=0,1, \ldots, T}, \quad \mathcal{F}_{T}=\mathcal{F}
$$

We assume that, for any $t=1,2, \ldots, T$, the measure $\mathrm{P}$ is complete on $\mathcal{F}_{t}$. Let $\bar{S}=$ $\left\{\bar{S}_{t}, t=0,1, \ldots, T\right\}$ be an $\mathcal{F}_{t}$-adapted and $(d+1)$-measurable process (a price vector). Let $S_{0}^{0}=1$ and $S_{t}^{0}=(1+r)^{t}$, where $r>0$ is a nonrandom interest rate.

Throughout this paper, except for Section 2, where we consider one-periodic models with random initial data, we assume that $\mathcal{F}_{0}=\{\varnothing, \Omega\}$.

A $(d+1)$-dimensional process

$$
\bar{\xi}=\left(\xi^{0}, \xi\right)=\left\{\left(\xi_{t}^{0}, \xi_{t}^{1}, \ldots, \xi_{t}^{d}\right), t=1, \ldots, T\right\}
$$

is called a strategy if it is $\mathcal{F}_{t}$-predictable, which means that the vector $\bar{\xi}_{t}$ is $\mathcal{F}_{t-1^{-}}$ measurable for all $t$.

$$
\begin{array}{r}
\text { A strategy } \bar{\xi}=\left\{\bar{\xi}_{t}, t=1,2, \ldots, T\right\} \text { is called self-financing if } \\
\bar{\xi}_{t} \cdot \bar{S}_{t}=\bar{\xi}_{t+1} \cdot \bar{S}_{t}, \quad t=1, \ldots, T-1 .
\end{array}
$$

Here and in what follows the symbol $x \cdot y$ stands for the scalar product of two vectors $x$ and $y$.

We define the discounted price process for the asset $S^{0}$ :

$$
X_{t}^{i}:=\frac{S_{t}^{i}}{S_{t}^{0}}, \quad t=0, \ldots, T, \quad i=0, \ldots, d .
$$

Put $\bar{X}_{t}:=\left(X_{t}^{0}, X_{t}\right)=\left(X_{t}^{0}, X_{t}^{1}, \ldots, X_{t}^{d}\right)$. A process

$$
V_{0}:=\bar{\xi}_{1} \cdot \bar{X}_{0}, \quad V_{t}:=\bar{\xi}_{t} \cdot \bar{X}_{t}, \quad t=1, \ldots, T,
$$

is called the discounted capital process for the strategy $\bar{\xi}$.

It is well known that

$$
V_{t}=V_{0}+\sum_{k=1}^{t} \xi_{k} \cdot \Delta X_{k}
$$

for a self-financing strategy $\bar{\xi}$, where $\Delta X_{k}=X_{k}-X_{k-1}$. 
The following definition is equivalent to the one introduced in [10].

Definition 1.1. A multiperiod model of a financial market admits an $\varepsilon$-arbitrage if there exists a self-financing strategy $\bar{\xi}=\left(\overline{\xi_{t}}\right)$ that is bounded in the sense that

$$
\sum_{t=0}^{T-1}\left\|\xi_{t}\right\|_{1}=\sum_{t=0}^{T-1} \sum_{i=1}^{d}\left|\xi_{t}^{i}\right| \leq 1 \quad \text { almost surely }
$$

and whose capital is such that

$$
V_{0} \leq 0, \quad \mathrm{P}\left\{V_{T} \geq \varepsilon\right\}=1, \quad \mathrm{P}\left\{V_{T}>\varepsilon\right\}>0 .
$$

Remark 1.2. It is easy to see that an $\varepsilon$-arbitrage is an arbitrage in a common sense, but the converse is not true. Thus an $\varepsilon$-arbitrage requires more from a process than a usual arbitrage. This feature distinguishes the notion of an $\varepsilon$-arbitrage from other generalizations (from an asymptotic arbitrage, for example, considered in [4, 3]).

We introduce a different but related definition. First we define the $\varepsilon$-capital of a self-financing strategy.

Definition 1.3. The process

$$
V_{t}^{\varepsilon}=V_{0}+\sum_{k=1}^{t} \xi_{k} \cdot \Delta X_{k}-\varepsilon \sum_{k=1}^{t}\left\|\xi_{k}\right\|_{1}
$$

is called the discounted $\varepsilon$-capital of a self-financing strategy $\bar{\xi}=\left(\overline{\xi_{t}}\right)$.

Remark 1.4. Definition 1.3 has a clear applied meaning. Frictionless models of a financial market considered in [1] allow investors to handle securities without restrictions, and this does not influence the prices in the market. The models with proportional transaction costs became popular recently (see, for example, [5]). In contrast to these models, the taxation in our model is proportional to the portfolio size and not to the costs of a transaction. If one speaks physics language, the friction in our model is caused by the weight (not by the movement).

Definition 1.5. A self-financing strategy $\bar{\xi}=\left\{\overline{\xi_{t}}, t=1,2, \ldots, T\right\}$ is called a $V^{\varepsilon}$-arbitrage if $\mathrm{P}\left(V_{T}^{\varepsilon} \geq 0\right)=1$ and $\mathrm{P}\left(V_{T}^{\varepsilon}>0\right)>0$.

Remark 1.6. At first glance, the notion of $V^{\varepsilon}$-arbitrage does not differ from that of $\varepsilon$ arbitrage (for a single period model, at least), since one can discount a strategy with its norm. Here is the problem though, since the norm may equal zero. In such a case, these notions are different, indeed. We demonstrate this feature by the following simple example.

Let $T=2, d=1, \Omega=\left\{\omega_{1}, \omega_{2}\right\}, \mathcal{F}_{1}=\mathcal{F}_{2}=2^{\Omega}, \mathrm{P}\left(\left\{\omega_{1}\right\}\right)=\mathrm{P}\left(\left\{\omega_{2}\right\}\right)=1 / 2, r=0$, $S_{0}^{1}=S_{1}^{1}\left(\omega_{1}\right)=S_{1}^{1}\left(\omega_{2}\right)=S_{2}^{1}\left(\omega_{1}\right)=1$, and $S_{2}^{1}\left(\omega_{2}\right)=1+2 \varepsilon$. Then $X_{1}=(0,0)$ and $X_{2}=(0,1+2 \varepsilon)$. It is obvious that an $\varepsilon$-arbitrage does not exist, since $V_{2}\left(\omega_{2}\right)=0$ for all strategies. On the other hand, the strategy $\xi_{1}=\xi_{2}\left(\omega_{1}\right)=0, \xi_{2}\left(\omega_{2}\right)=1$ obviously is a $V^{\varepsilon}$-arbitrage.

We prove in Section 3 that the above definition of $V^{\varepsilon}$-arbitrage is equivalent to the following one.

Definition 1.7. A strategy $\left\{\bar{\xi}_{t}, t=1, \ldots, T\right\}$ is called $\varepsilon$-self-financing if

$$
\bar{\xi}_{t+1} S_{t}=\bar{\xi}_{t}-(1+r)\left\|\xi_{t}\right\|_{1} \text {. }
$$

Such a strategy is called a $K^{\varepsilon}$-arbitrage if

$$
\mathrm{P}\left(K_{T} \geq 0\right)=1, \quad \mathrm{P}\left(K_{T}>0\right)>0,
$$


where

$$
K_{t}=\bar{\xi}_{t} \cdot \bar{S}_{t}
$$

is the capital related to the strategy $\xi$.

There are two reasons for introducing this definition of $\varepsilon$-self-financing strategy. First, a tax applies immediately after a portfolio is closed (this explains the factor $1+r$ with the tax). Second, the tax is due to the risky assets, while the operations with nonrisky assets are nontaxable.

\section{ONE-PERIOD MODEL WITH RANDOM INITIAL DATA}

Let $T=1$, that is, we deal with a one-period model of a financial market. We do not assume that $\mathcal{F}_{0}$ is trivial, and this allows us to consider random initial data. Put $\Delta X=X_{1}-X_{0}$ and $\|x\|_{\infty}=\max _{i}\left|x^{i}\right|$.

For the sake of completeness, we first provide a result from [9, 10] for a one-period model of a financial market.

Lemma 2.1 (9]). The following conditions are equivalent:

1) a one-period financial market admits an $\varepsilon$-arbitrage;

2) there exists an $\mathcal{F}_{0}$-measurable process $\xi$ such that $\|\xi\|_{1} \leq 1$ almost surely and $\xi \cdot \Delta X \geq \varepsilon$ almost surely with respect to $\mathrm{P}$. Moreover $\mathrm{P}\{\xi \cdot \Delta X>\varepsilon\}>0$.

Theorem 2.2 ([10]). The following conditions are equivalent for a one-period financial market:

1) the market is $\varepsilon$-arbitrage-free;

2) there exists a probability measure $\mathrm{P}^{*}$ equivalent to $\mathrm{P}$ and such that $d \mathrm{P}^{*} / d \mathrm{P} \leq C$ for some constant $C<\infty$ and

$$
\mathrm{E}_{\mathrm{P}^{*}}\left[\left\|\mathrm{E}_{\mathrm{P} *}\left[\Delta X / \mathcal{F}_{0}\right]\right\|_{\infty}\right] \leq \varepsilon .
$$

A natural question arises on whether this result can be generalized to a norm in the space $\mathbb{R}^{d}$ that is different from $\|\cdot\|_{1}$. More precisely, let $\|\cdot\|_{\sim}$ be a norm in $\mathbb{R}^{d}$ and let $\|\cdot\|_{\sim}^{*}$ be its conjugate norm. An $\mathcal{F}_{0}$-measurable strategy $\xi \in \mathbb{R}^{d}$ is called an $\varepsilon \sim$-arbitrage strategy for the vector $X \in \mathbb{R}^{d}$ in a one-period model if $\|\xi\|_{\sim} \leq 1, \xi \cdot X \geq \varepsilon$ almost surely, and $\mathrm{P}(\xi \cdot X>\varepsilon)>0$. A partial generalization of Theorem 2.2 can be found in [10].

Theorem 2.3 ([10]). For all $\delta>0$,

1) a one-period model is $(\varepsilon+\delta) \sim$-arbitrage-free;

2) there exists a measure $\mathrm{P}^{*} \sim \mathrm{P}$ with a bounded density and such that

$$
\mathrm{E}_{\mathrm{P}^{*}}\left[\left\|\mathrm{E}_{\mathrm{P} *}\left[\Delta X / \mathcal{F}_{0}\right]\right\|_{\sim}^{*}\right] \leq \varepsilon+\delta .
$$

Is it possible to drop $\delta$ in Theorem 2.3? It is clear that the existence of an equivalent measure $\mathrm{P}^{*} \sim \mathrm{P}$ such that $\mathrm{E}_{\mathrm{P}^{*}}\left[\left\|\mathrm{E}_{\mathrm{P} *}\left[\Delta X / \mathcal{F}_{0}\right]\right\|_{\sim}\right] \leq \varepsilon$ implies the nonexistence of an $\varepsilon \sim-$ arbitrage. Unfortunately, the converse statement is not true in general. We demonstrate this in the following example.

Example 2.4. Let $d=2$ and let $\|\xi\|_{\sim}=|\xi|=\left(\left(\xi^{1}\right)^{2}+\left(\xi^{2}\right)^{2}\right)^{1 / 2}$ be the usual Euclidean norm. Define the probability space by $\Omega=\left\{\omega_{1}, \omega_{2}\right\}, \mathcal{F}_{0}=\{\varnothing, \Omega\}, \mathcal{F}_{1}=2^{\Omega}$, and

$$
\mathrm{P}\left(\left\{\omega_{1}\right\}\right)=\mathrm{P}\left(\left\{\omega_{2}\right\}\right)=\frac{1}{2} .
$$

Let the discounted price vector be given by $X_{0}=(0,0), X_{1}\left(\omega_{1}\right)=(1,0)$, and $X_{1}\left(\omega_{2}\right)=$ $(1,2)$. Then this model is 1 -arbitrage-free. Indeed, if $|\xi|=1$ and $\xi \cdot \Delta X \geq 1$ almost 
surely, then $\xi^{1} \geq 1$ (for $\omega=\omega_{1}$ ), since $\xi=(1,0)$. Then $\xi \cdot X\left(\omega_{2}\right)=1$, whence the nonexistence of an $\varepsilon \sim$-arbitrage follows. On the other hand,

$$
\mathrm{E}_{\mathrm{P}^{*}}\left[\left|\mathrm{E}_{\mathrm{P}^{*}}\left[\Delta X \mid \mathcal{F}_{0}\right]\right|\right]=\left|\mathrm{E}_{\mathrm{P}^{*}}[\Delta X]\right|=\left|\left(1,2 p^{*}\right)\right|>1
$$

for $\mathrm{P}^{*} \sim \mathrm{P}$ such that $p^{*}=\mathrm{P}^{*}\left(\left\{\omega_{2}\right\}\right) \in(0,1)$.

One can prove similarly that the implication 2 ) $\Rightarrow 1$ ) of Theorem 2.2 does not hold for the norms $\|\cdot\|_{p}$ and, in general, for any norms whose balls are convex at a certain point $a$ in the following sense: there exists a unique hyperplane whose intersection with that ball contains a single point $a$. This consideration leads to the conjecture below. Unfortunately, the author is not aware of a proof of it or of a counterexample to it.

Conjecture 2.5. Suppose a ball of a norm $\|\cdot\|_{\sim}$ is not convex at any point. Then the following conditions are equivalent:

1) the one-period model is E -arbitrage-free;

2) there exists a measure $\mathrm{P}^{*} \sim \mathrm{P}$ with bounded density and such that

$$
\mathrm{E}_{\mathrm{P}^{*}}\left[\mathrm{E}_{\mathrm{P}^{*}}\left\|\left[\Delta X / \mathcal{F}_{0}\right]\right\|_{\sim}^{*}\right] \leq \varepsilon .
$$

Now we consider a $V^{\varepsilon}$-arbitrage.

Note that a $V^{\varepsilon}$-arbitrage is equivalent to an $\varepsilon$-arbitrage for a one-period model with nonrandom initial data. In view of this remark, the following result claims the same as Lemma 3.6 does in [10]. We denote by $\overline{\mathbb{R}}^{d}$ a single point compactification of the space $\mathbb{R}^{d}$. We treat $C\left(\overline{\mathbb{R}}^{d}\right)$ as a normed space equipped with the norm $\|g\|_{\text {sup }}=\max _{\overline{\mathbb{R}}^{d}}|g|$.

Lemma 2.6. Assume that there is no $V^{\varepsilon}$-arbitrage for a random vector $Y \in \mathbb{R}^{d}$ with a distribution $P_{Y}(d x)$. This means that if $\xi \cdot Y \geq \varepsilon\|\xi\|_{1}$ almost surely for $\xi \in \mathbb{R}^{d}$, then $\xi \cdot Y=\varepsilon\|\xi\|_{1}$ almost surely. Then there exists a positive function $g \in C\left(\overline{\mathbb{R}}^{d}\right)$ such that

$$
\int_{\mathbb{R}^{d}} g(y) P_{Y}(d y)=1, \quad\left\|\int_{\mathbb{R}^{d}} y g(y) P_{Y}(d y)\right\|_{\infty} \leq \varepsilon .
$$

This result is used for the proof of the existence of an equivalent "martingale" measure in the case of random initial data in a way similar to that in the proof in [1.

Theorem 2.7. The following conditions are equivalent:

1) a financial market is $V^{\varepsilon}$-arbitrage-free;

2) there exists a measure $\mathrm{P}^{*}$ equivalent to $\mathrm{P}$ and such that $d \mathrm{P}^{*} / d \mathrm{P} \leq C$ for some $C>0$ and $\left\|\mathrm{E}_{\mathrm{P}^{*}}\left[\Delta X / \mathcal{F}_{0}\right]\right\|_{\infty} \leq \varepsilon$ almost surely.

Proof. $[2) \Rightarrow 1)]$ Let $\xi \in \mathbb{R}^{d}$ be an $\mathcal{F}_{0}$-measurable vector such that

$$
\mathrm{P}\left(\xi \cdot \Delta X \geq \varepsilon\|\xi\|_{1}\right)=1 .
$$

Then

$$
\begin{aligned}
\mathrm{E}_{\mathrm{P}^{*}}\left[\xi \cdot \Delta X-\varepsilon\|\xi\|_{1}\right] & =\mathrm{E}_{\mathrm{P}^{*}}\left[\mathrm{E}_{\mathrm{P}^{*}}\left[\xi \cdot \Delta X-\varepsilon\|\xi\|_{1} / \mathcal{F}_{0}\right]\right] \\
& \leq \mathrm{E}_{\mathrm{P}^{*}}\left[\mathrm{E}_{\mathrm{P}^{*}}\left[\|\xi\|_{\infty}\|\Delta X\|_{1} / \mathcal{F}_{0}\right]-\varepsilon\|\xi\|_{1}\right] \\
& \leq \mathrm{E}_{\mathrm{P}^{*}}\left[\|\xi\|_{1}\left(\mathrm{E}\left[\|\Delta X\|_{\infty} / \mathcal{F}_{0}\right]-\varepsilon\right)\right] \leq 0 .
\end{aligned}
$$

Thus $\xi \cdot \Delta X=\varepsilon\|\xi\|_{1}$ almost surely with respect to both $\mathrm{P}^{*}$ and $\mathrm{P}$. Therefore a $V^{\varepsilon_{-}}$ arbitrage does not exist.

$[1) \Rightarrow 2)]$ We introduce an equivalent measure $\mathrm{P}^{\prime} \sim \mathrm{P}$ with a bounded density by putting $d \mathrm{P}^{\prime} / d \mathrm{P}=C /\left(1+\|\Delta X\|_{1}\right)$, where $C$ is a normalizing constant. The vector $\Delta X$ is integrable with respect to $\mathrm{P}^{\prime}$. Moreover, there is no $V^{\varepsilon}$-arbitrage with respect to $\mathrm{P}^{\prime}$, and every measure that is equivalent to $P^{\prime}$ and has a bounded density with respect to $P^{\prime}$ 
is also equivalent to $\mathrm{P}$ and has a bounded density with respect to $\mathrm{P}$. Without loss of generality we assume that $\mathrm{E}\left[\|\Delta X\|_{1}\right]<\infty$.

Denote by $\mathrm{P}(\omega, d x)$ the regular conditional distribution of $\Delta X$ given $\mathcal{F}_{0}$. For vectors $\alpha \in \mathbb{R}^{d}$, we put $H_{\alpha \varpi}^{\varepsilon}=\left\{x: \alpha \cdot x \varpi \varepsilon\|x\|_{1}\right\}$, where $\varpi$ means one of the signs $\{\leq,<,=,>, \geq\}$.

We consider the set $\mathfrak{A}$ where the $V^{\varepsilon}$-arbitrage is attained, namely

$$
\mathfrak{A}=\left\{(\omega, x) \in \Omega \times \mathbb{R}^{d}: \mathrm{P}\left(\omega, H_{x \geq}^{\varepsilon}\right)=1, \mathrm{P}\left(\omega, H_{x>}^{\varepsilon}\right)>0\right\} .
$$

This set belongs to $\mathcal{F}_{0} \otimes \mathcal{B}\left(\mathbb{R}^{d}\right)$, since the functions $\mathrm{P}\left(\omega, H_{x \geq}^{\varepsilon}\right)$ and $\mathrm{P}\left(\omega, H_{x>}^{\varepsilon}\right)>0$ are $\mathcal{F}_{0} \otimes \mathcal{B}\left(\mathbb{R}^{d}\right)$-measurable (see Lemma 4.1).

Then the projection of $\mathfrak{A}$,

$A=\operatorname{Pr}_{\Omega} \mathfrak{A}=\left\{\omega:\right.$ there exists $x \in \mathbb{R}^{d}$ such that $\mathrm{P}\left(\omega, H_{x \geq}^{\varepsilon}\right)=1$ and $\left.\mathrm{P}\left(\omega, H_{x>}^{\varepsilon}\right)>0\right\}$, belongs to $\mathcal{F}_{0}$ by the projection theorem 8 .

In view of the measurable choice theorem, there exists an $\mathcal{F}_{0}$-measurable random vector $\zeta \in \mathbb{R}^{d}$ such that $(\omega, \xi) \in \mathfrak{A}$ for almost all $\omega \in A$. The vector $\zeta=\xi \mathbb{1} A$ is $\mathcal{F}_{0}$-measurable and

$$
\begin{aligned}
\mathrm{P}(\zeta \cdot \Delta X \geq \varepsilon\|\zeta\|) & =\mathrm{E}\left[\mathrm{E}\left[\mathbb{1} \zeta \cdot \Delta X \geq \varepsilon\|\zeta\| / \mathcal{F}_{0}\right]\right]=\mathrm{E}\left[\mathrm{P}\left(\omega, H_{\zeta \geq}^{\varepsilon}\right)\right] \\
& =\mathrm{E}\left[\mathbb{1} A \mathrm{P}\left(\omega, H_{\xi \geq}^{\varepsilon}\right)+\mathbb{1} A^{c} \mathrm{P}\left(\omega, H_{0 \geq}^{\varepsilon}\right)\right]=1 .
\end{aligned}
$$

Analogously,

$$
\mathrm{P}(\zeta \cdot \Delta X \geq \varepsilon\|\zeta\|)=\mathrm{E}\left[\mathbb{1} A \mathrm{P}\left(\omega, H_{\xi>}^{\varepsilon}\right)\right] .
$$

Since there is no $V^{\varepsilon}$-arbitrage and since the probability $\mathrm{P}\left(\omega, H_{\xi>}^{\varepsilon}\right)$ is positive for $\omega \in A$, we have $\mathrm{P}(A)=0$.

Now we consider the set

$$
\mathfrak{H}=\left\{(\omega, g) \in \Omega \times C\left(\overline{\mathbb{R}}^{d}\right): g \geq 0, \int_{\mathbb{R}^{d}} g(x) \mathrm{P}(\omega, d x)=1, \int_{\mathbb{R}^{d}} x g(x) \mathrm{P}(\omega, d x) \in[-\varepsilon, \varepsilon]^{d}\right\} .
$$

It belongs to $\mathcal{F}_{0} \otimes \mathcal{B}\left(C\left(\overline{\mathbb{R}}^{d}\right)\right)$. Since $\mathrm{P}(A)=0$ and there is no $V^{\varepsilon}$-arbitrage, we get, for almost all $\omega$, that an $V^{\varepsilon}$-arbitrage does not exist for a random vector with the distribution $\mathrm{P}(\omega, d x)$. By Lemma 2.6 a function $g_{\omega} \in C\left(\overline{\mathbb{R}}^{d}\right)$ exists for almost all $\omega$ and is such that $\left(\omega, g_{\omega}\right) \in \mathfrak{H}$. In other words, the projection of $\mathfrak{H}$ on $\Omega$ is of full probability. Since $C\left(\overline{\mathbb{R}}^{d}\right)$ is a complete separable space, the measurable choice theorem implies that there exists an $\mathcal{F}_{0}$-measurable function $G: \Omega \rightarrow C\left(\overline{\mathbb{R}}^{d}\right)$ such that $(\omega, G(\omega)) \in \mathfrak{H}$ almost surely. The mapping $(\omega, x) \rightarrow G(\omega, x):=G(\omega)(x)$ is $\mathcal{F}_{0} \otimes \mathcal{B}\left(\mathbb{R}^{d}\right)$-measurable as a composition of measurable mappings. Thus $D(\omega):=M(\omega) G(\omega, Y(\omega))$ is $\mathcal{F}_{1}$-measurable, where

$$
M(\omega)=1 /(1+K(\omega)), \quad K(\omega)=\|G(\omega, \cdot)\|_{\text {sup }} .
$$

Note that

$$
\mathrm{E}[G(\omega, Y(\omega))]=\mathrm{E}\left[\mathrm{E}\left[G(\omega, Y(\omega)) / \mathcal{F}_{0}\right]\right]=\mathrm{E}\left[\int_{\mathbb{R}^{d}} g(\omega, x) \mathrm{P}(\omega, d x)\right]=1
$$

and

$$
M(\omega)=\mathrm{E}\left[D(\omega) / \mathcal{F}_{0}\right]
$$

Now we define $d \mathrm{P}^{*}(\omega)=M D(\omega) d \mathrm{P}(\omega)$, where $M=1 / \mathrm{E}[D(\omega)]$. Then

$$
\begin{aligned}
\left\|\mathrm{E}_{\mathrm{P}^{*}}\left[Y / \mathcal{F}_{0}\right]\right\|_{\infty} & =\left\|\mathrm{E}\left[Y(\omega) D(\omega) / \mathcal{F}_{0}\right] / \mathrm{E}\left[D(\omega) / \mathcal{F}_{0}\right]\right\|_{\infty} \\
& =\left\|\mathrm{E}\left[Y(\omega) G(\omega, Y(\omega)) / \mathcal{F}_{0}\right]\right\|_{\infty} \\
& =M \mathrm{E}\left[D(\omega)\left\|\int_{\mathbb{R}^{d}} x G(\omega, x) \mathrm{P}(\omega, d x)\right\|_{\infty}\right] \leq \varepsilon .
\end{aligned}
$$




\section{MAIN THEOREM FOR A MULTIPERIOD MODEL}

Put

$$
R_{T}^{\varepsilon}:=\left\{V_{T}^{\varepsilon}: V_{T}^{\varepsilon}=\sum_{t=1}^{T} \xi_{t} \cdot \Delta X_{t}-\varepsilon \sum_{t=1}^{T}\left\|\xi_{t}\right\|_{1}\right\},
$$

where $\xi$ is an arbitrary self-financing strategy. This means that $R_{T}^{\varepsilon}$ is the set of all possible results arising from a zero initial capital under the $\varepsilon$-tax proportional to the portfolio size.

Put $A_{T}^{\varepsilon}:=R_{T}^{\varepsilon}-L_{+}^{0}=\left\{V-V^{+}: V \in R_{T}^{\varepsilon}, V^{+} \in L_{+}^{0}\right\}$. Here $L_{+}^{0}$ is the set of all finite nonnegative random variables defined on the probability space $(\Omega, \mathcal{F}, \mathrm{P})$.

Theorem 3.1. The following conditions are equivalent:

1) the model is $V^{\varepsilon}$-arbitrage-free, that is, $\mathbb{R}_{T}^{\varepsilon} \cap L_{+}^{0}=\{0\}$;

2) for all $t=1,2, \ldots, T$, the one-period model is $V_{\varepsilon}$-arbitrage-free in the interval $[t-1, t]$ (this means that if $\xi \in \mathbb{R}^{d}$ is an $\mathcal{F}_{t-1}$-measurable vector and

$$
\mathrm{P}\left(\xi \cdot \Delta X_{t} \geq \varepsilon\|\xi\|_{1}\right)=1,
$$

then $\xi \cdot \Delta X_{t}=\varepsilon\|\xi\|_{1}$ almost surely);

3) the model is $K^{\varepsilon}$-arbitrage-free;

4) $A_{T}^{\varepsilon} \cap L_{+}^{0}=\{0\}$;

5) there exists a measure $\mathrm{P}^{*} \sim \mathrm{P}$ with bounded density such that $\left\|\mathrm{E}_{\mathrm{P}^{*}}\left[\Delta X_{t} / \mathcal{F}_{t-1}\right]\right\|_{\infty}$ $\leq \varepsilon$ for all $t=1,2, \ldots, T$.

Remark 3.2. The classical result of Dalang, Morton, and Willinger [1] follows from Theorem 3.1 with $\varepsilon=0$.

In contrast to the analogous result for the classical notion of arbitrage (see [6]), we cannot claim that the set $A_{T}^{\varepsilon}$ is closed if (4) holds. The proof of the classical case is reduced to the proof of orthogonality of certain vectors and the assumption that $\varepsilon=0$ plays a crucial role here. An attempt to repeat the classical proof in the general case gives that the cosine of an angle between certain vectors is equal to $\varepsilon$ on a certain set of positive probability, and one cannot conclude anything from this fact. It would be interesting to find another proof of the closedness property mentioned above.

Proof. The implication [1) $\Rightarrow$ 2)] is obvious.

The implication (4) $\Rightarrow$ 10] follows from $R_{T}^{\varepsilon} \subset A_{T}^{\varepsilon}$.

The implication [5) $\Rightarrow$ 2)] follows from Theorem 2.7 .

Now we prove [2) $\Rightarrow[1]$ ]. We show that the existence of a $V^{\varepsilon}$-arbitrage in such a model implies the existence of a $V^{\varepsilon}$-arbitrage in one of the periods. Let $\left\{\xi_{t}\right\}$ be a strategy corresponding to the $V^{\varepsilon}$-arbitrage. Put

$$
t_{0}=\min \left\{t: \mathrm{P}\left(V_{t}^{\varepsilon} \geq 0\right)=1, \mathrm{P}\left(V_{t}^{\varepsilon}>0\right)>0\right\} .
$$

This definition is correct because a $V^{\varepsilon}$-arbitrage exists. Since the number $t_{0}$ is minimal, we conclude that either $V_{t_{0}-1}^{\varepsilon}=0$ almost surely, and then, obviously, $\xi_{t_{0}}$ corresponds to a $V^{\varepsilon}$-arbitrage for the period $\left[t_{0}, t_{0}-1\right]$, or $\mathrm{P}\left(V_{t_{0}-1}^{\varepsilon}=0\right)<1$, and then $\xi_{t_{0}} \mathbb{1} V_{t_{0}-1}^{\varepsilon}<0$ is a $V^{\varepsilon}$-arbitrage strategy.

Consider the equivalence (1) $\Leftrightarrow$ 3)]. We construct a correspondence between the selffinancing strategy (with its $\varepsilon$-capital) and an $\varepsilon$-self-financing strategy (and its capital). This will mean the equivalence between $V^{\varepsilon}$ - and $K^{\varepsilon}$-arbitrages.

It is easy to check by induction that the discounted capital of an $\varepsilon$-self-financing strategy $\left\{\bar{\xi}_{t}=\left(\xi_{t}^{0}, \xi_{t}\right)\right\}$ is equal to

$$
V_{t}=(1+r)^{-t} K_{t}=V_{0}+\sum_{k=1}^{t} \xi_{k} \cdot \Delta X_{k}-\sum_{k=1}^{t}\left\|\xi_{k}\right\|_{1} .
$$


This process coincides with the $\varepsilon$-capital of the self-financing strategy that uniquely corresponds to $\left\{\xi_{t}\right\}$. On the other hand, if a self-financing strategy $\left\{\bar{\xi}_{t}=\left(\xi_{t}^{0}, \xi_{t}\right)\right\}$ exists, then we consider another strategy $\left\{\bar{\zeta}_{t}=\left(\zeta_{t}^{0}, \xi_{t}\right)\right\}$ with $\zeta_{t}^{0}=\xi_{t}^{0}-\sum_{k=1}^{t}\left\|\xi_{t}\right\|_{1}$. It is easy to check that the latter strategy is $\varepsilon$-self-financing and its discounted capital coincides with the $\varepsilon$-capital of the strategy $\bar{\xi}$.

Now we check the implication [1) $\Rightarrow$ (4)]. Assume that condition (1) holds but condition (4) does not. In other words, there exists a nonzero $z \in A_{T}^{\varepsilon} \cap L_{+}^{0}$. Then there exist $\zeta \in R_{T}^{\varepsilon}$ and $u \in L_{+}^{0}$ such that

$$
z=\zeta-u \geq 0 \Rightarrow \zeta \geq u \geq 0 \Rightarrow \zeta \geq 0 \Rightarrow \zeta=0,
$$

since condition (1) implies that $R_{T}^{\varepsilon} \cap L_{+}^{0} \subset\{0\}$, whence we conclude that $\zeta$ is equal to zero. In its turn, the equality $\zeta=0$ implies that $u=0$, whence $z=0$. This contradiction proves the implication [10 $\Rightarrow$ 4)].

Finally we prove the implication $[21) \Rightarrow[5]$ ]. If condition (2) holds, then there is no $V^{\varepsilon}$-arbitrage on the interval $[t-1, t]$ for all $t=1,2, \ldots, T$.

We use a backward induction to construct a measure $\mathrm{P}^{*}$; that is, we construct a measure $\mathrm{P}_{t} \sim \mathrm{P}$ with bounded density and such that

$$
\left\|\mathrm{E}_{\mathrm{P}_{t}}\left[\Delta X_{s} / \mathcal{F}_{s-1}\right]\right\|_{\infty} \leq \varepsilon \text { for } s=t+1, t+2, \ldots, T .
$$

Put $\mathrm{P}_{T}=\mathrm{P}_{0}$. Suppose the measure $\mathrm{P}^{*}$ is constructed for a certain $t$. Since there is no $V^{\varepsilon}$-arbitrage in the interval $[t-1, t]$ with respect to the measure $\mathrm{P}$, and since $\mathrm{P}_{t} \sim \mathrm{P}$, a $V^{\varepsilon}$-arbitrage does not exist with respect to the measure $\mathrm{P}$ either. Then Theorem 2.7 implies that there exists a measure $\mathrm{P}_{t-1} \sim \mathrm{P}_{t}$ with a bounded and $\mathcal{F}_{t}$-measurable density $d \mathrm{P}_{t-1} / d \mathrm{P}_{t}$ such that $\left\|\mathrm{E}\left[\Delta X_{t} / \mathcal{F}_{t-1}\right]\right\|_{\infty} \leq \varepsilon$. Moreover, we have, for $s \geq t+1$, that

$$
\begin{aligned}
\mathrm{E}_{\mathrm{P}_{t-1}}\left[\Delta X_{s} / F_{s-1}\right] & =\mathrm{E}_{\mathrm{P}_{t}}\left[\frac{d \mathrm{P}_{t-1}}{d \mathrm{P}_{t}} \Delta X_{s} / F_{s-1}\right] / \mathrm{E}\left[\frac{d \mathrm{P}_{t-1}}{d \mathrm{P}_{t}} / F_{s-1}\right] \\
& =\frac{d \mathrm{P}_{t-1}}{d \mathrm{P}_{t}} \mathrm{E}_{\mathrm{P}_{t}}\left[\Delta X_{s} / F_{s-1}\right] / \frac{d \mathrm{P}_{t-1}}{d \mathrm{P}_{t}}=\mathrm{E}_{\mathrm{P}_{t}}\left[\Delta X_{s} / F_{s-1}\right],
\end{aligned}
$$

where we have used the measurability with respect to $\mathcal{F}_{t}$ which implies the $\mathcal{F}_{s-1^{-}}$ measurability of $d \mathrm{P}_{t-1} / d \mathrm{P}_{t}$. By the induction assumption, $\mathrm{P}_{t} \sim \mathrm{P}$ and $d \mathrm{P}_{t} / d \mathrm{P}$ is bounded; thus $\mathrm{P}_{t-1} \sim \mathrm{P}$ and

$$
\frac{d \mathrm{P}_{t-1}}{d \mathrm{P}}=\frac{d \mathrm{P}_{t-1}}{d \mathrm{P}_{t}} / \frac{d \mathrm{P}_{t}}{d \mathrm{P}}
$$

is bounded.

Finally, we set $\mathrm{P}^{*}=\mathrm{P}_{0}$ and obtain a measure for which condition (5) holds, and this completes the proof of the theorem.

\section{A LEMma on Joint MEASURABility of THE REgular EXPECTATion}

Lemma 4.1. Let a function $f(x, y): \mathbb{R}^{k} \times \mathbb{R}^{d} \rightarrow \mathbb{R}^{m}$ be measurable and let $B \in \mathcal{B}\left(\mathbb{R}^{m}\right)$. Assume that $P(\omega, d y)$ is the regular distribution of a random vector $\xi \in \mathbb{R}^{d}$ with respect to the $\sigma$-algebra $\mathcal{G} \subset \mathcal{F}$.

Then the function $g_{f}(\omega, x)=P(\omega,\{y: f(x, y) \in B\})$ is $\mathcal{G} \otimes \mathcal{B}\left(\mathbb{R}^{d}\right)$-measurable.

Proof. It suffices to prove, for $C \in \mathcal{B}\left(\mathbb{R}^{k} \times \mathbb{R}^{d}\right)$, that the function

$$
g_{C}(\omega, x)=P(\omega,\{y:(x, y) \in C\})
$$

is $\mathcal{G} \otimes \mathcal{B}\left(\mathbb{R}^{d}\right)$-measurable.

Let $\mathcal{C}=\left\{C: g_{C}\right.$ is $\mathcal{G} \otimes \mathcal{B}\left(\mathbb{R}^{d}\right)$-measurable $\}$. We prove that $\mathcal{C} \supset \mathcal{B}\left(\mathbb{R}^{k} \times \mathbb{R}^{d}\right)$.

1. First we check that $\mathcal{C}$ is a Dynkin system. If $C^{\prime}, C^{\prime \prime} \in \mathcal{C}$ and $C^{\prime} \subset C^{\prime \prime}$, then $C^{\prime \prime} \backslash C^{\prime} \in \mathcal{C}$. Indeed, $g_{C^{\prime \prime} \backslash C^{\prime}}=g_{C^{\prime \prime}}-g_{C^{\prime}}$. 
If $C_{n} \in \mathcal{C}$ and $C_{n} \subset C_{n+1}$ for all $n$, then $\bigcup_{n \geq 1} C_{n} \in \mathcal{C}$. Indeed,

$$
g_{C}=\lim _{n \rightarrow \infty} g_{C_{n}} .
$$

2. Note that $X \times Y \in \mathcal{C}$ for all open sets $X \subset \mathbb{R}^{k}$ and $Y \subset \mathbb{R}^{d}$. Indeed,

$$
g_{C}(\omega, x)=P(\omega, Y) \mathbb{1} X(x)
$$

is $\mathcal{G} \otimes \mathcal{B}\left(\mathbb{R}^{d}\right)$-measurable. The class $\mathcal{O}$ of all the sets of this form is closed with respect to finite intersections, and thus it is a $\pi$-system.

Therefore $\mathcal{C} \supset \sigma(\mathcal{O})=\mathcal{B}\left(\mathbb{R}^{k} \times \mathbb{R}^{d}\right)$ by Dynkin's theorem, and this is what was to be proved.

\section{Concluding Remarks}

The notion of $V^{\varepsilon}$-arbitrage (in other words, of an arbitrage for a model where there is a taxation on the portfolio size) is considered in this paper. The studies of this and some related notions require mathematical tools different from the classical ones, since some of $V^{\varepsilon}$-arbitrage-free financial markets may have an arbitrage in the classical meaning.

The results obtained in 9 provide the main conditions that are equivalent to the criteria of nonexistence of a bounded arbitrage in a one-period financial market. Those results are generalized to one-period models with random initial data and to multiperiod models in the paper [10]. Similar results are proved in the present paper, but for a different setting. This new setting also has a clear practical interpretation and allows one to obtain sharper results than those in [10].

The main result of this paper is similar to that of the classical arbitrage theory and gives a relationship between the existence of a bounded arbitrage and the existence of a measure with certain properties. In contrast to the classical case, where the measure is martingale, the measure is $\varepsilon$-martingale in our setting: $\left|\mathrm{E}_{\mathrm{P}^{*}}\left[\Delta X_{t}^{i} / \mathcal{F}_{t-1}\right]\right| \leq \varepsilon$ for all $i$

and $t$ (here $\Delta X_{t}^{i}$ is the discounted increment of the price of an $i$ th asset in the interval $[t-1, t])$. This condition becomes the classical arbitrage-free condition if $\varepsilon=0$.

\section{Acknowledgement}

The author is grateful to Professor F. Nazarov, University of Wisconsin-Madison, USA, for his suggestions concerning the proof of Lemma 4.1.

\section{BIBLIOGRAPHY}

1. R. C. Dalang, A. Morton, and W. Willinger, Equivalent martingale measures and no-arbitrage in stochastic securities market models, Stochastics Stochastics Rep. 29 (1990), 185-201. MR.1041035 (91g:90056)

2. F. Delbaen and W. Schachermayer, A general version of the fundamental theorem of asset pricing, Math. Ann. 300 (1994), 463-520. MR1304434 (95m:90022b)

3. H. Föllmer and W. Schachermayer, Asymptotic arbitrage and large deviations, Finance Stoch. 1 (1997), no. 3, 181-227. MR2403770 (2009c:91046)

4. Yu. Kabanov and D. Kramkov, Asymptotic arbitrage in large financial markets, Finance Stoch. 2 (1998), no. 2, 143-172. MR.1806101 (2001m:91090)

5. Yu. Kabanov and M. Safarian, Markets with Transaction Costs, Springer Finance, Springer, Berlin, 2009. MR 2589621

6. Yu. M. Kabanov and Ch. Stricker, A teachers' note on no-arbitrage criteria, Séminaire de Probabilités XXXV, Lect. Notes Math., vol. 1755, Springer, Berlin, 2001, pp. 149-152. MR1837282 (2003c:60073)

7. J. Liu and F. Longstaff, Losing money on arbitrages: optimal dynamic portfolio choice in markets with arbitrage opportunities, Rev. Financial Studies 71 (2004), no. 3, 611-641.

8. C. Dellacherie, Capacités et processus stochastiques, Springer-Verlag, Berlin-New York, 1972. MR0448504(56:6810) 
9. Yu. S. Mishura, The main theorem of financial mathematics for a bounded arbitrage, Applied Statistics. Actuarial and Finance Mathematics 2003, no. 1-2, 49-54. (Ukrainian)

10. Yu. S. Mishura, G. M. Shevchenko, and P. S. Shelyazhenko, Limited arbitrage in a multiperiod model of a financial market with discrete time, Teor. Imovir. Mat. Stat. 77 (2007), 122-131; English transl. in Theory Probab. Math. Statist. 77 (2008), 135-146. MR2432777|(2009c:91074)

11. H. Föllmer and A. Schied, Stochastic Finance. An Introduction in Discrete Time, Walter de Gruyter \& Co., Berlin, 2002. MR.1925197 (2004h:91051)

12. A. N. Shiryaev, Essentials of Stochastic Finance. Facts, Models, Theory, Fazis, Moscow, 1998; English transl., World Scientific, River Edge, NJ, 1999. MR1695318 (2000e:91085)

Department of Probability Theory, Statistics, and Actuarial Mathematics, Faculty for Mechanics and Mathematics, National Taras Shevchenko University, Academician Glushkov Avenue 2, Kiev 03127, Ukraine

E-mail address: zhora@univ.kiev.ua

Received 28/SEP/2009

Translated by S. KVASKO 